\title{
Unscented Kalman Filter for Unobservable Parameter Estimation in Heart Cell Signals
}

\author{
David Adolfo Sampedro-Puente ${ }^{1}$, Jesus Fernandez-Bes ${ }^{2,1}$, Esther Pueyo ${ }^{1,2}$ \\ ${ }^{1}$ Biomedical Signal Interpretation and Computational Simulation (BSICoS) \\ Instituto de Investigación en Ingeniería de Aragón (I3A) \\ Universidad de Zaragoza, Mariano Esquillor s/n, 50018, Zaragoza, Spain. \\ Tel. +34-976762707, e-mail: sampedro@unizar.es \\ ${ }^{2}$ CIBER-BBN: Centro de Investigación Biomédica en Red - Bioingeniería, Biomateriales y Nanomedicina.
}

\begin{abstract}
One interesting feature of biological systems is that minor subcellular changes can cause alterations at the whole organ level. In the heart, the random dynamics of cell membrane ion channels contributes to beat-to-beat repolarization variability, which has been related to proarrhythmic risk. Inference of unobservable cellular parameters, such as the number of channels, is key to characterize such random ion channel dynamics. In this work, a methodology based on the use of Unscented Kalman Filters is proposed to infer the number of channel from action potential signals, like those commonly recorded experimentally.
\end{abstract}

\section{Introduction}

In recent years, there has been a growing interest in the use of computational models in cadiac research to complement experimental and clinical trials [1]. In particular, a variety of models to characterize the electrophysiological behavior of heart cells both for human and animals have been proposed [2]. Nevertheless, the vast majority of these models are deterministic, i.e., they provide identical responses for each simulated beat. Consequently, they cannot be used to simulate variability in the action potencial (AP).

Beat-to-beat variability (also known as temporal variability) is related to proarrhythmic risk [3]. When observed at a single myocyte scale this variability has been associated to the randomness in ion channel gating. The use of stochastic computational models, by allowing a characterization of electrophysiological variability, may help in the understanding of proarrhythmia mechanisms and in the design of specific clinical therapies.

This work proposes a novel approach based on Unscented Kalman Filters (UKF) [4] to infer unobservable parameters characteristic of cardiomyocyte behavior. Synthetic AP signals generated by a stochastic model are used to assess the performance of the proposed approach.

\section{Materials and Methods}

Human Ventricular Stochastic Model

In this work, a two-current model based on the formulations of the Ten Tusscher-Panfilov (TP06) human ventricular cell model has been implentented [5]. Specifically, this model represents only the rapid and slow delayed rectifier potassium currents ( $\mathrm{I}_{\mathrm{KR}}$ and $\mathrm{I}_{\mathrm{KS}}$, respectively). Stochasticity is added into the equations describing the gating dynamics of these two currents by considering stochastic differential equations (SDE) of Langevin type driven by Wiener noise [6]. Using this approach, the stochastic term for each ion channel gate is inversely proportional to the square root of the number of ion channels of that type. Synthetic AP signals are generated with the proposed stochastic model, where the number of ion channels is set to $\mathrm{N}_{\text {IKS }}=\mathrm{N}_{\text {IKR }}=11000$, based on results from previous studies [6,7]. A train of 300 beats corresponding at $1 \mathrm{~Hz}$ pacing is simulated using this stochastic model.

\section{Unscented Kalman Filter}

The previous two-current model in SDE is rewritten as a state-space model composed by 5 state variables and 2 parameters. These variables are the action potential (AP), the potassium concentration inside the cell $\left(\mathrm{K}_{\mathrm{i}}\right)$, the two different types of $\mathrm{I}_{\mathrm{KR}}$ gates $\left(X_{R 1}\right.$ and $\left.X_{R 2}\right)$ and finally the $I_{K S}$ gate $\left(X_{S}\right)$. The number of $\mathrm{I}_{\mathrm{KR}}$ channels and $\mathrm{I}_{\mathrm{KS}}$ channels $\left(\mathrm{N}_{\mathrm{IKR}}\right.$ and $\mathrm{N}_{\mathrm{IKS}}$, respectively) are the two parameters of this state-space model. In this model, the only measured variable is the AP and we assume that it is measured under Gaussian noise with zero mean and unit variance.

In this work, the UKF method is chosen to jointly track these state variables and estimate the parameters from a noisy synthetic AP signal simulated by the previous stochastic model. 


\section{Results}

The first two figures show that the UKF methodology is able to estimate the different variables of the space-state model. Fig. 1 illustrates a 4-cycle-length AP. As we can observe in this figure, the UKF method provides a suitable estimation of the AP only from a synthetic AP signal measured under noise conditions.

The second figure shows a 4-cycle-length sequence of the gate variable $X_{R 1}$. As we can observe in this figure, the results calculated by UKF method are quite similar to the values obtain by stochastic model simulation, with the additional gain of an estimated uncertaintly band provided by the UKF.

Finally, Fig. 3 points out that UKF approach is also valid to track variations in the number of channels. In this scenario, the $\mathrm{N}_{\text {IKR }}$ of the stochastic simulation changes from 11000 to 14000 , and UKF is able to find a suitable estimation of the number of $\mathrm{I}_{\mathrm{KR}}$ ion channels.

Similarly, the results for $I_{K S}$ ion channels are in concordance with $I_{K R}$ results, for that reason only figures with the $\mathrm{I}_{\mathrm{KR}}$ outcomes are shown.

\section{Conclusion}

The promising results shown in this work demonstrate the capability of the proposed UKF approach to estimate unobservable cell parameters, specifically the number of ion channel, from a synthetic AP signal simulated by a stochastic model with a determined setup.

Future works will involve the processing of real AP signals obtained from human biopsies to infer the number of ion channels presents in the different real cardiomyocytes.

\section{References}

[1]. MEURS, W.V. Modeling and Simulation in Biomedical Engineering: Applications in Cardiorespiratory Physiology. 2011. ISBN 0071714456.

[2]. NOBLE, D., GARNY, A. and NOBLE, P.J. How the Hodgkin-Huxley equations inspired the Cardiac Physiome Project. The Journal of physiology. 1 Jun 2012. Vol. 590, no. 11, p. 2613-28.

[3]. VARKEVISSER, R., WIJERS, S.C., VAN DER HEYDEN, M.A.G., BEEKMAN, J.D.M., MEINE, M. and VOS, M.A. Beat-to-beat variability of repolarization as a new biomarker for proarrhythmia in vivo. Heart rhythm: the official journal of the Heart Rhythm Society . Oct 2012. Vol. 9, no. 10, p. 1718-26.

[4]. JULIER, S.J. and UHLMANN, J.K. A new Extension of the Kalman Filter to Nonlinear Systems. AeroSense'97. Proc. of AeroSense: The 11th Int. Symp. on
Aerospace/Defence Sensing. Simulation and Controls. 28 Jul 1997. p. 182-193

[5]. TEN TUSSCHER, K.H.W.J. and PANFILOV, A.V. Alternans and spiral breakup in a human ventricular tissue model. American journal of physiology. Heart and circulatory physiology. Sep 2006. Vol.291, no. 3, p. H1088-100.

[6]. PUEYO, E., CORRIAS, A., VIRÁG, L., JOST, N., SZÉL, T., VARRÓ, A., SZENTANDRÁSSY, N., NÁNÁSI, P.P, BURRAGE, K. and RODRÍGUEZ, B. A multiscale investigation of repolarization variability and its role in cardiac arrhythmogenesis. Biophysical journal. 21 December 2011. Vol. 101, no. 12, p. 2892-902.

[7]. VELDKAMP, M.W., VAN GINNEKEN, A.C., OPTHOF, T. and BOUMAN, L.N. Delayed rectifier channels in human ventricular myocytes. Circulation. 15 Dec 1995. Vol. 92, no. 12, p. 3497-504.

\section{Acknowledgments:}

This work is supported by personal grant to D. A. Sampedro-Puente and by Grupo Consolidado BSICoS (T96) from Government of Aragón (Spain) and European Social Fund (EU), by project TIN2013-41998-R from MINECO and by CIBER-BBN through ISCIII. The computation was performed by the ICTS NANBIOSIS, by the High Performance Computing Unit of the CIBER-BBN at the University of Zaragoza.

\section{FIGURES}

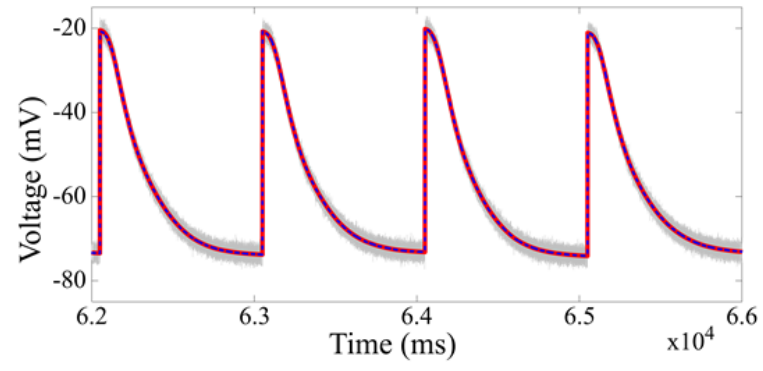

Figure 1. AP used as measured variable (gray line), measured AP without noise (red line) and AP obtained by UKF method (blue dashed line).

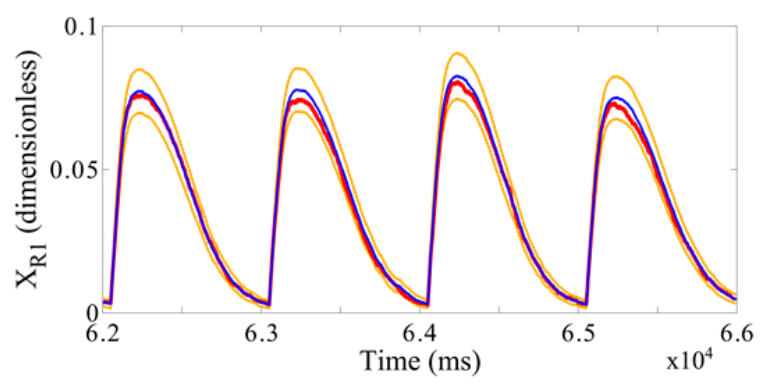

Figure 2. $X_{\mathrm{R} 1}$ gate obtained by stochastic model (red line) and the mean (blue) \pm 3 times standard desviation (orange) of the estimated parameter obtained by UKF.

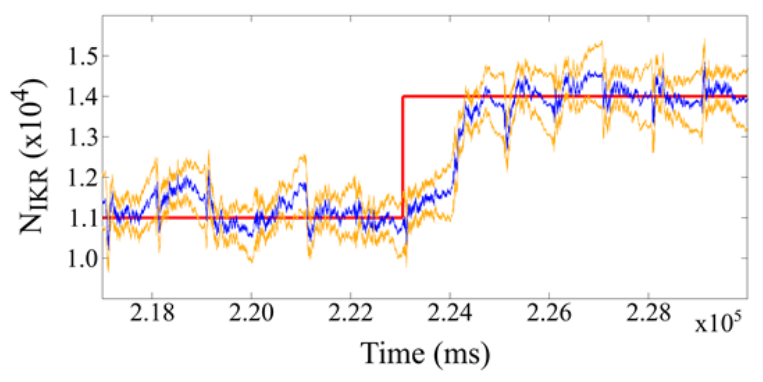

Figure 3. Simulated number of $I_{K R}$ channels (red line) and the mean (blue) \pm standard desviation (orange) of the estimated parameter obtained by UKF. 